\title{
Yolk-sac hematopoiesis: The first blood cells of mouse and man
}

\author{
James Palis ${ }^{\mathrm{a}}$ and Mervin C. Yoder ${ }^{\mathrm{b}}$ \\ ${ }^{a}$ University of Rochester, Department of Pediatrics and Cancer Center, Rochester, NY, USA; \\ ${ }^{\mathrm{b}}$ Indiana University School of Medicine, Herman B. Wells Center for Pediatric Research, Indianapolis, IN, USA
}

\begin{abstract}
Objective. To review the process of blood-cell formation in the murine and human yolk sac. Data Sources. Most articles were selected from the PubMed database.

Data Synthesis. The yolk sac is the first site of blood-cell production during murine and human ontogeny. Primitive erythroid cells originate in the yolk sac and complete their maturation, including enucleation, in the bloodstream. Though species differences exist, the pattern of hematopoietic progenitor cell emergence in the yolk sac is similar in mouse and man. In both species, there is a stage of development where both primitive red blood cells and definitive erythroid progenitors are produced in the yolk sac. An "embryonic" hematopoietic stem cell that engrafts in myeloablated newborn but not adult mice can be detected in the murine yolk sac and embryo. Stem-cell activity in the human yolk sac has not been reported.

Conclusions. The yolk sac is the sole site of embryonic erythropoiesis. However, definitive erythroid, myeloid, and multipotential progenitors also originate in the yolk sac. The relationship between these progenitors and the "embryonic" hematopoietic stem cell has not been elucidated. Yolk sac-derived progenitor cells may seed the developing liver via the circulation and serve as the immediate source of the mature blood cells that are required to meet the metabolic needs of the rapidly growing fetus. () 2001 International Society for Experimental Hematology. Published by Elsevier Science Inc.
\end{abstract}

In the adult, hematopoiesis is in a steady state as all formed elements of the blood are synthesized from multipotent precursor cells produced by hematopoietic stem cells in the bone marrow. In the fetus, the liver serves as the principal hematopoietic organ. However, the early mammalian embryo requires red cells for growth and survival prior to development of the liver. Large primitive red cells are produced in blood islands of the yolk sac soon after implantation of the embryo. Primitive red cells have marked differences from the definitive red cells later produced in the fetal liver and postnatal bone marrow [1]. A recent review in this journal has discussed the development of the hematopoietic system in the mouse embryo, comparing it to hematopoiesis observed in cultured murine embryonic stem cells [2]. Here we focus on the cell biology of hematopoiesis in the yolk sac, including a more detailed description of primitive erythropoiesis and a discussion of the origins of hematopoi-

Offprint requests to: James Palis, M.D., University of Rochester, Department of Pediatrics, Box 777, Rochester, NY 14642; E-mail: james_palis@urmc.rochester.edu etic stem cells. A brief overview of human yolk-sac hematopoiesis is also presented.

Yolk-sac hematopoiesis in the mouse

\section{The first blood and endothelial}

cells arise in yolk-sac blood islands

The murine yolk sac is a bilayer organ composed of extraembryonic mesoderm cells apposed to visceral endoderm cells. This association of cells derived from mesoderm and endoderm germ layers is termed splanchnopleure. Normal embryonic development is critically dependent on the proper functioning of the yolk sac since its endoderm layer transports and metabolizes maternally-derived macromolecules and synthesizes serum proteins, and its mesoderm layer produces the first blood cells within blood islands (reviewed by [3]). Furthermore, the visceral endoderm is thought to serve as a source of inductive signals important for the formation of blood cells [4,5] and endothelial networks [6] in the yolk sac. GATA-4-null embryonic stem cells form embryoid bodies lacking visceral endoderm and also display markedly reduced blood-island formation, further supporting the con- 
cept that the visceral endoderm participates in the development and organization of blood islands [7]. Mesoderm/endoderm recombination experiments suggest that these signals act during early gastrulation and are diffusible [5].

Yolk-sac blood islands were first observed in the early 1900's [8]. Detailed light and electron-microscope studies have been performed in several mammalian species, including mouse $[9,10]$ and human (see below). In the mouse, presumptive blood islands, known as mesodermal cell masses or angioblastic cords, arise from proximal mesodermal cells in the visceral yolk sac between E7 and E7.5 (Fig. 1). Blood islands are bordered by visceral endoderm cells facing the yolk-sac cavity and the single cell layer of mesothelium facing the exocoelomic cavity (Fig. 1). Between E8.0 and E9.0, the cells comprising the outer layer of the blood-island cell aggregates assume a spindle shape as they differentiate into endothelial cells. The vast majority of the inner cells progressively lose their intercellular attachments as they differentiate into primitive erythroblasts [9].

The synchronous temporal and intimate spatial appearance of blood cells and endothelial cells in yolk-sac blood islands has led to the concept of a common "hemangioblast" precursor for the blood and endothelial lineages. Recent studies with cells from chick embryos and with embryonic stem (ES) cells have led credence to this concept. In the avian embryo, mesoderm cells expressing the vascular endothelial growth factor (VEGF) receptor-2 (flk-1) differentiate in vitro into endothelial cells in the presence of VEGF or into hematopoietic cells in the absence of VEGF [11]. Murine ES cells cultured as embryoid bodies in vitro contain blast colony-forming cells that form both endothelial and hematopoietic cells upon secondary replating [12]. The absence of yolk-sac blood islands in mutant mouse embryos lacking $f l k-1$ provides further evidence suggesting that endothelial cells and blood cells originate from a common precursor cell [13]. However, no in vivo proof of a clonal rela-

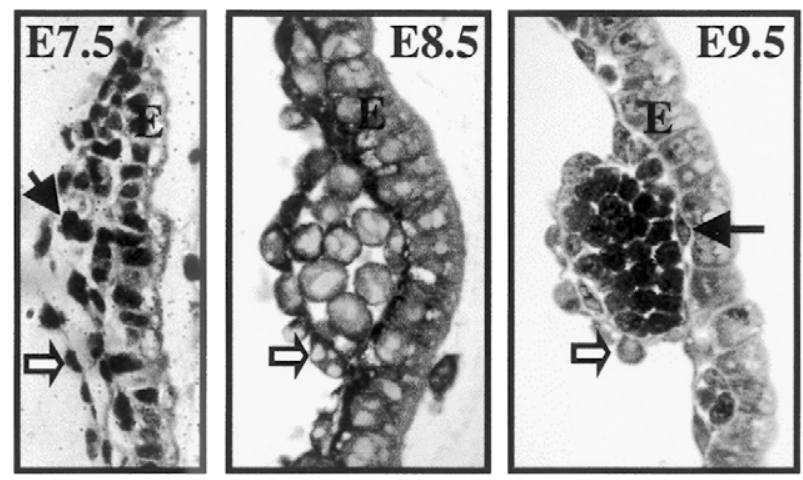

Figure 1. Development of yolk sac blood islands in the mouse embryo between E7.5 and E9.5. Yolk sac blood islands form between the single cell layer of endoderm cells (E) and mesothelial cells (open arrow). Blood islands develop from undifferentiated mesoderm cells (E7.5, closed arrow) that give rise to inner blood cells surrounded by an outer endothelial lining (E9.5, closed arrow). tionship between endothelial cells and hematopoietic cells has yet been presented.

Primitive erythropoiesis originates in the yolk sac

Soon after their initial development in yolk-sac blood islands, primitive erythroblasts enter the newly formed vasculature of the embryo proper. There they continue to divide for several days (Fig. 2), as evidenced by mitotic cells in the bloodstream [14], thymidine incorporation studies [15], and cell-cycle analysis [16]. Primitive erythroblasts differentiate within the bloodstream, gradually accumulating increasing amounts of hemoglobin and becoming progressively less basophilic [10]. Hemoglobin synthesis directed by stable globin transcripts continues until cell replication ceases $[17,18]$. Primitive murine erythroblasts continue to divide until E13, at which point they reach their steady-state hemoglobin content of $80 \mathrm{pg} / \mathrm{cell}$, nearly four times the amount of hemoglobin found in adult erythrocytes [19].

Initial studies using electrophoretic methods to separate hemoglobins indicated that primitive erythroblasts and adult erythrocytes express different hemoglobins and that adulttype hemoglobin accumulation coincides with the appearance of enucleated fetal liver-derived erythrocytes [20]. Subsequent studies of separated globin chains confirmed the finding that primitive erythroblasts initially synthesize embryonic globins and indicated that they later synthesize adult globins [8,21]. This switch in globin synthesis occurs in all yolk-sac erythroblasts examined by specific antibodies directed against embryonic and adult hemoglobin [21].

Primitive murine erythroblasts attain a mean cell volume of 465-530 fL ([22]; Palis, unpublished data), earning for them the name of megaloblasts. As megaloblasts differentiate in the bloodstream, there is progressive nuclear condensation [20]. Enucleated "megalocytes" having the same size and hemoglobin content as nucleated yolk-sac erythroblasts are first evident at E13 [14]. These cells are thought to be derived from yolk-sac erythroblasts that have undergone enucleation, since they are threefold larger than the fetal liver-

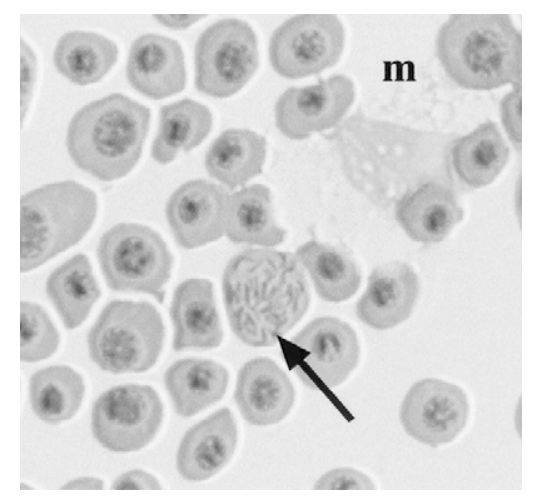

Figure 2. Cytospin preparation of circulating mouse blood cells at E10.5. The vast majority of cells are differentiating primitive erythrobasts, some still in mitosis (arrow). Rare macrophage cells (m) are also present. 
derived "macrocytes" that enter the bloodstream beginning at E12 [19]. While nucleated yolk sac-derived erythroid cells are no longer present in the bloodstream after E16 [23], megalocytes continue to circulate until E18 [14]. Primitive erythroblasts also undergo other changes during differentiation consistent with enucleation, including the loss of the intermediate filament vimentin [16] and loss of histone proteins [24]. These observations indicate that terminal differentiation of primitive erythroid cells results in erythrocytes more similar to the enucleated red cells of mammals rather than the nucleated red cells of birds and fish.

Erythropoiesis in the bone marrow is critically dependent on the growth factor erythropoietin, which is thought to be both a mitogen and survival factor for mature definitive erythroid progenitors [25]. However, the role of erythropoietin in yolk-sac erythropoiesis remains controversial. Early studies indicated that the addition of exogenous erythropoietin did not affect heme synthesis when gastrulating mouse embryos were cultured intact [26]. These results have been widely interpreted to indicate that primitive erythropoiesis is not dependent on erythropoietin. However, in a subsequent study by the same investigator, exogenous erythropoietin caused a significant increase in heme synthesis when added to cultures of disaggregated embryonic cells [27]. Disaggregated E8.5 yolk-sac cells cultured for 18 hours in the presence of erythropoietin contain significantly more hemoglobin $^{+}$cells and significantly fewer dead cells compared to cultures lacking exogenous erythropoietin [28]. These effects of erythropoietin (increasing erythroid cell numbers and preventing cell death) were not evident when circulating yolk-sac erythroblasts from E9.5 and E10.5 mouse embryos were examined [28]. Other studies have also shown that the addition of exogenous erythropoietin to yolk sacs explanted from E7.5-E8.5 mouse embryos results in increased numbers of primitive erythroid cells and in increased accumulation of embryonic globin transcripts [29,30]. These findings indicate that yolk-sac erythroid cells respond to erythropoietin and suggest that erythropoietin may act as a survival factor for primitive erythroblasts early in their maturation.

Proof that erythropoietin signaling has a functional role in primitive erythropoiesis comes from the targeted disruption of several murine genes, particularly erythropoietin, the erythropoietin receptor, and the intracellular signaling molecule Jak2 kinase. In each case, there is a fivefold to 20 -fold reduction in number of circulating primitive erythroblasts by E11.5 and the mutant embryos die with severe anemia by E13.5 [31-34]. However, the persistence of some hemoglobin-containing primitive erythroblasts in the circulation of mutant embryos is in striking contrast to the complete lack of terminally differentiated definitive erythroid cells in the fetal liver. The relative independence of primitive erythropoiesis from erythropoietin signaling raises the unanswered question of what other cytokine signaling cascades are active in yolk-sac erythroblasts.
Consistent with a role for erythropoietin in the differentiation of primitive red cells, erythropoietin receptor transcripts are present at the earliest stages of yolk-sac bloodisland formation in the mouse [30]. Hamster yolk-sac erythroblasts initially express increasing numbers of erythropoietin receptors on their cell surface as they circulate in the bloodstream [35]. Receptor numbers subsequently decrease as terminal differentiation proceeds. While erythropoietin transcripts have been detected in the mouse embryo at E7.5 and in the fetal liver at E14.5, the low levels of erythropoietin expression have hampered the spatial and temporal analysis of its expression during development [36-38]. A small percentage $(7 \%-10 \%)$ of a supraphysiologic dose of erythropoietin can cross the mouse placenta just prior to birth (E19) [39]. However, it is not known if physiologic levels of maternal erythropoietin cross the murine placenta, or if maternal erythropoietin can cross the yolk sac, particularly prior to formation of the chorioallantoic placenta, which occurs between E8.5 and E9.5.

The ability to culture hematopoietic progenitors in semisolid medium has contributed significantly to our understanding of the hematopoietic hierarchy in the adult. Early erythroid progenitors in the murine bone marrow are recognized by the generation of large colonies of red cells in methylcellulose (burst-forming unit erythroid; BFU-E) following 7-10 days in culture, while more mature erythroid progenitors generate smaller colonies (colony-forming unit erythroid; CFU-E) within 2-3 days in culture. Wong et al. identified a unique intermediate erythroid progenitor type during gastrulation in the mouse embryo $[29,40]$. These progenitor colonies, called "day-5 CFU-E," consisted of approximately 100 red cells expressing both embryonic and adult globins. Day-5 CFU-E progenitors arose early on the eighth gestational day exclusively in the yolk sac and disappeared by E9 [40]. It was subsequently confirmed that day 5 CFU-E are primitive erythroid progenitors (EryP-CFC) that generate colonies in semisolid media with a distinct timing of differentiation, morphology, and pattern of gene expression compared to the definitive BFU-E and CFU-E [41]. EryP-CFC generate colonies comprised of large nucleated primitive erythroid cells that express both embryonic and adult globins. In contrast, BFU-E and CFU-E give rise to colonies comprised of small definitive red cells expressing only adult globins [42].

A more extensive analysis of the development of hematopoietic progenitors in the early postimplantation mouse embryo has recently been carried out [42]. Using carefully staged embryos, it was determined that the first EryP-CFC arise at $\mathrm{mid} /$ late primitive streak stages (E7.0), within hours of the start of mesoderm formation (Fig. 3). The spatial distribution of these progenitors within the conceptus and their subsequent expansion in numbers correlated with the formation of extraembryonic mesoderm cells in the visceral yolk sac. EryP-CFC are confined to the yolk sac, where they expand in numbers at E8.25 (early somite pair stages). However, by E9.0 (20-25 somite pair stages) they were no longer 


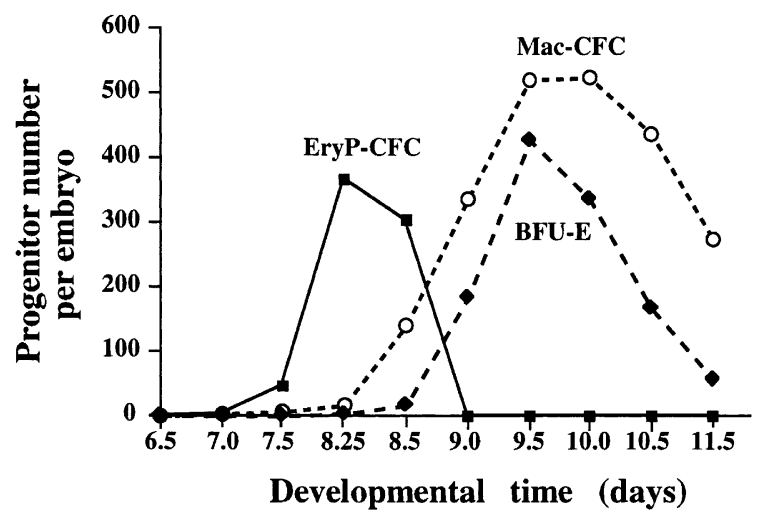

Figure 3. Development of primitive erythroid (EryP-CFC), definitive erythroid (BFU-E), and macrophage (Mac-CFC) progenitors in the mouse yolk sac between E6.5 and E11.5 of gestation. Data derived from Palis et al. [42].

present in the conceptus ([40,42] and Fig. 3). These studies indicate that primitive erythroid progenitor production is confined to the yolk sac and primitive erythropoiesis constitutes the only transient lineage during hematopoietic ontogeny.

\section{Definitive erythropoiesis is initiated in the yolk sac}

Initial studies of definitive erythroid progenitors in the mouse embryo indicated that BFU-E were first detected at E8.5 and CFU-E at E9.5 [43]. Wong et al. [40] confirmed that BFU-E arose late on the eighth day of gestation and continued to increase in number at E9 and E10. Unexpectedly, these definitive erythroid progenitors were found exclusively in the yolk sac at E8.5, but by E9.0 were also present in the bloodstream and the embryo proper [40,44]. Recently, an analysis of carefully staged mouse embryos revealed that BFU-E originate in the yolk sac of early somite pair embryos at E8.25, just prior to the onset of circulation [42]. Over the subsequent 24 hours, BFU-E increase in numbers within the yolk sac (Fig. 3) and enter the newly formed bloodstream of the embryo proper. CFU-E are subsequently found in the yolk sac, the bloodstream, and the embryo proper, consistent with their differentiation from yolk sac-derived BFU-E. Between E10.5 and E11.5, the number of BFU-E and CFU-E decline in the yolk sac and expand exponentially within the liver rudiment [42].

The fact that definitive erythrocytes are first evident at E12, when they begin their egress from the fetal liver, suggests that the yolk sac-derived definitive erythroid progenitors (BFU-E and CFU-E) cannot fully differentiate within the yolk-sac environment. This conclusion is supported by explant experiments using tissues obtained from 20-25 somite pair mouse embryos [45]. Yolk sacs explanted alone contain a transient wave of erythroid cells expressing embryonic and adult globins, consistent with the presence of primitive erythroid cells. In contrast, yolk-sac tissues cocultured adjacent to liver primordium revealed a subsequent wave of yolk-sac erythroid cells expressing adult globins. These results indicate that definitive erythroid progenitors are present in the yolk sac and that factors in the embryo proper, but not the yolk sac, support their differentiation. Taken together, these studies indicate that both primitive and definitive erythropoiesis originate in the yolk sac. While the transient wave of primitive erythroid progenitors remains confined to the yolk sac, the second wave of definitive erythroid progenitors enters the circulation and likely seeds the liver primordium beginning at E9.5.

Multiple myeloid lineages originate and expand in the yolk sac The developmental biology of the macrophage lineage has been recently reviewed in this journal [46] and will be discussed only briefly here. The first macrophage cells identified in the mouse embryo are visible as clusters of free ameboid cells with distinct nucleoli in the yolk sac between E9 and 9.5 [47]. Genes associated with the macrophage lineage, including the macrophage colony-stimulating factor (M-CSF) receptor c-fms and F4/80, are first detected in the yolk sac and the head of mouse embryos between E9.0 and E9.5 [48,49]. Unlike tissue macrophages in the adult, which are thought to derive from monocyte cells, embryonic macrophages appear to directly differentiate from macrophage progenitors.

In pioneering work, Moore and Metcalf determined that macrophage progenitors (Mac-CFC) are present in intact presomite embryos and in the yolk sac of early somite pair embryos [50], consistent with the progenitors arising in the yolk sac. A recent detailed analysis identified macrophage progenitors in mid to late primitive streak embryos at E7.0 in the distal regions of the embryo associated with the developing yolk sac [42]. After an initial expansion within the yolk sac (Fig. 3), macrophage progenitors are subsequently found in the bloodstream and throughout the embryo proper.

The yolk sac is also the site of origin of microglial progenitors that form large myeloid colonies in vitro when plated in culture with astrogrial monolayers. These microglial precursors were first detected at presomitic stages of development (E7.0) within the yolk sac and subsequently at early stages of somite development (E8.0) in the neural folds [51]. The results of this kinetic analysis suggest, but do not prove, that microglial precursors migrate directly from the yolk sac into the developing central nervous system. These results are consistent with studies performed in the chick, where microglial precursors migrate from the yolk sac to the developing central nervous system (CNS) without circulating in the bloodstream [52]. Upon reaching the central nervous system, the yolk sac-derived microglial precursors proliferate extensively, to eventually comprise the entire population of mature microglial cells in the adult animal. It is not known if these microglial progenitor cells are distinct from the Mac-CFC detected in the yolk sac [42,50].

Mast cell and granulocyte/macrophage (CFU-GM) progenitors are first detected in the yolk sac of the 10-15 somite pair mouse conceptus [42]. These progenitors initially expand in the yolk sac [53], but are subsequently found in higher numbers in the bloodstream and liver of the 
fetus, consistent with their migration into the embryo proper from the yolk sac [42]. Thus, like the primitive and definitive erythroid lineages, multiple myeloid lineages also originate and expand in numbers within the yolk sac between E7.0 and 10.5.

\section{Multipotential hematopoietic \\ progenitors are first detectable in the yolk sac}

In the adult, committed hematopoietic progenitors arise from multipotential precursors. The ordered appearance in the yolk sac of two distinct erythroid and several myeloid progenitor types suggests that the yolk sac contains multipotential hematopoietic precursors. The spleen colony-forming assay (CFU-S) and high proliferative colony-forming assay (HPP$\mathrm{CFC}$ ) have been used to determine the presence of multipotential precursors in the early mouse embryo. The former relies on the homing of hematopoietic precursors to the spleen of a lethally irradiated adult recipient, while the latter generates a macroscopic colony of myeloid cells in agar that is dependent on the presence of multiple growth factors [54]. Moore and Metcalf [50] first detected CFU-S8 at E8.5 in the yolk sac but not the embryo proper, suggesting that multipotential precursors arise in the yolk sac. In contrast, Medvinsky et al. [55] did not detect CFU-S8 until E9.5 (26-27 somite pairs), when they were simultaneously identified in the yolk sac and embryo proper. It is not clear what factors are responsible for these discordant findings.

HPP-CFC have been detected as early as E8.0-E8.5 in the mouse conceptus; however, the spatial distribution of these precursors in staged embryos was not examined [56]. We have recently conducted an extensive analysis of the spatial and temporal emergence of HPP-CFC in the mouse embryo [57]. HPP-CFC were not found at presomite stages (E6.5-E7.5), despite examining more than 180 embryos. The first HPP-CFC were detected at early somite stages (E8.25), exclusively in the yolk sac. The yolk sac remains the predominant site of HPP-CFC expansion until E11.5, when the liver supplants the yolk sac as the primary site of hematopoietic progenitor production. These findings indicate that HPP-CFC originate in the yolk sac after the onset of primitive erythropoiesis and suggest that multipotential hematopoietic precursors emerge from the yolk sac concomitant with the definitive erythroid and several myeloid lineages.

\section{The origin of lymphoid precursors remains controversial} Lymphoid cells normally mature during the second half of gestation within the fetal liver and thymus of the embryo proper. The developmental origin of the lymphoid progenitors that seed these organs remains controversial. Several investigators have examined both yolk-sac and embryoproper tissues for the presence of lymphoid potential. Most of the studies have found the simultaneous appearance of lymphoid progenitors in both yolk sac and embryo proper [58-61]. However, other studies have indicated that lym- phoid potential arises within the embryo proper between E8.5 and E9.5 [62,63] and even earlier if the tissues are first explanted and expanded for several days on a bone marrow stromal cell line [64]. Other investigators have focused exclusively on either the yolk sac or the AGM region as the source of lymphoid potential $[65,66]$. Ultimately, the source of lymphoid precursors is likely linked to the embryologic origin of hematopoietic stem cells (HSC).

\section{"Embryonic" hematopoietic stem cells arise before "adult" hematopoietic stem cells}

All blood cells are postulated to derive from HSC. These stem cells represent a largely quiescent population that demonstrate self-renewal, replenish circulating blood cells in transplanted recipients, and are resistant to 5-fluorouracil treatment. Studies in the mouse embryo have indicated that HSCs able to engraft adult mice are present in the liver beginning at E11-E12 [67-69]. These fetal liver-derived HSCs express CD34, c-kit, AA4.1, and Sca-1 surface markers, and are thought to migrate to the bone marrow after E15 [70,71]. Since the liver rudiment is colonized by exogenous blood cells [72], HSCs must arise elsewhere. It was initially hypothesized that HSCs originate in the yolk sac, migrate to the liver, and eventually seed the bone marrow [50]. However, the yolk sac does not contain HSCs before E11.5 that are capable of engrafting adult recipients [53,67].

This stem-cell model was further challenged by studies in the avian embryo, where yolk-sac cells do not contribute to long-term adult hematopoiesis (reviewed by [73]). In the avian embryo, foci of hematopoietic cells, found initially in intra-aortic regions, and later in para-aortic clusters, are responsible for long-term erythropoiesis in the adult. Morphologic evidence of intra-aortic cell clusters has also been found in several mammalian species, including the mouse, pig, gerbil, and human [74,75]. Investigations in the mouse embryo indicate that that HSCs capable of engrafting adult recipients are associated with the aorta in the AGM region at E10-E10.5, one day before their appearance in the liver and yolk sac $[68,76]$.

The sine qua non of HSC function is the repopulation of the hematopoietic system of myeloablated recipients following transplantation. For adult recipients, this entails repopulation of the bone marrow. An alternative explanation for the lack of HSC activity prior to mid-gestation is that HSCs do, in fact, exist but are unable to engraft in the adult hematopoietic microenvironment. Evidence that HSCs exist prior to E10 comes from transplant studies using mouse embryos as recipients. E9.0 yolk sac and peripheral blood cells were used to engraft E11-E15 W/W host embryos, which are HSC deficient [77]. While there was tremendous fetal loss caused by the cell injections, 6/163 embryonic recipients showed evidence of long-term engraftment of the erythroid lineage. Unfortunately, donor embryos were not staged, embryo-proper tissues were not studied, and only erythroid engraftment was measured. Weissman et al. [78] injected E8- 
E10 yolk-sac cells into E8-E9 donor embryos. Low levels of donor lymphoid cells were identified after birth of the recipients. Again, donor embryos were not staged and embryoproper cells were not investigated. However, these studies provide evidence suggesting that "embryonic" HSCs exist in the pre-E10 mouse embryo and that these HSCs are capable of homing to fetal hematopoietic organs [79].

More recently, a newborn recipient model of transplantation has been developed [80]. Rationale for these experiments encompassed several principles of developmental hematopoiesis. From E12 until shortly after birth, the murine liver is the predominant site of HSC residence. Though the fetal marrow compartment is seeded with HSC at day E15, the liver retains the majority of HSC activity at birth [81]. Thus, in contrast to the adult recipient in which only bone marrow and spleen are sites for HSC engraftment, the newborn recipient presents the liver, bone marrow, and spleen as potential hematopoietic engraftment sites. However, transplantation of hematopoietic cells into unablated newborn or fetal mice generally fails to result in readily detectable and durable engraftment. Use of mice genetically deficient in HSC activity has been the most successful strategy for achieving donor-cell engraftment in fetal or newborn recipients (see above).

Busulfan is one of several chemotherapeutic agents that is known to cross the placenta and cause untoward effects on fetal development. We [80] determined that certain doses of busulfan could be administered to pregnant mice without fetal wastage. Pregnant mice were treated two days prior to birth with busulfan to reduce endogenous hematopoiesis in the fetuses. Donor cells were injected directly into the livers or intravenously into the facial vein of the newborn pups and donor contribution to peripheral blood cells was monitored for 4-6 months to ensure long-term hematopoietic engraftment. In initial experiments, cells from E10 yolk sacs were able to provide long-term multilineage reconstitution of busulfan-conditioned newborns [82]. Subsequent studies have shown that embryonic HSCs expressing CD34 and c-kit reside both in the yolk sac and in the developing AGM region at E9.0 [83]. A 37-fold preponderance of E9.0 yolk-sac cells expressing CD34 and c-kit suggests, but does not prove, that embryonic HSCs arise in the yolk sac. Tissues derived from earlier developmental stages, particularly prior to the onset of the circulation, will need to be investigated to determine the temporal and spatial origin of embryonic HSCs.

Not only did embryonic HSCs provide long-term engraftment of newborn recipients, but bone marrow cells from these primary recipients at 4-6 months posttransplant engrafted in lethally irradiated secondary adult recipients [84]. These results demonstrate that embryonic HSCs can become "adult" HSCs. At present, little is known of the molecules that effect the change from embryonic to "adult" HSC. The contribution of embryonic HSCs to hematopoiesis in the adult and the relationship of embryonic HSCs to their adult counterparts found later in the AGM region and fetal liver remain to be elucidated. This information is crucial if one desires to utilize primary embryonic HSC or hematopoietic precursors derived in vitro from embryonic stem cells for transplant into adult recipients.

\section{Yolk-sac hematopoiesis in man}

The primary sites of hematopoiesis also change in a temporally and spatially ordered fashion during human development. Blood cells first arise in the yolk sac, followed sequentially by the fetal liver and finally the developing long bones $[85,86]$. The yolk sac serves as the initial site of erythropoiesis from weeks $3-6$ of gestation. The liver functions as the primary site of hematopoiesis from weeks 6-22 of gestation, after which the bone marrow becomes the predominant and lifelong site of blood-cell production. Distinct patterns of blood-cell differentiation and proliferation are noted in each specific site of hematopoiesis during ontogeny [87-89]. The ontogeny of blood-cell production in the human embryo has been described using both morphologic approaches and in vitro hematopoietic progenitor cell assays. We will briefly review the formation and function of the human yolk sac, the kinetics of blood-cell appearance, and the ontogeny of hematopoietic progenitor cells in the human yolk sac.

\section{Yolk-sac formation differs in man and mouse}

The human yolk sac develops in three general phases: 1) a formative period, 2) a functional period, and 3) a period of regression [90]. The first phase can be further subdivided into formation of the primary and secondary yolk sac. The primary yolk sac is formed soon after implantation by proliferation and differentiation of primitive endoderm cells into visceral and parietal endoderm. Some controversy surrounds the identification of the cell lineage from which the first yolk-sac mesoderm cells emerge in the human embryo. In contrast to the mouse embryo, where yolk-sac mesoderm cells are derived from primitive ectoderm (epiblast) during gastrulation, yolk-sac mesoderm cells are thought to arise from primitive endoderm (hypoblast) in the primate embryo (reviewed in [90]). In the rhesus monkey, extraembryonic mesoderm cells appear to delaminate from the parietal endoderm of the hypoblast. Emergence of human and rhesus yolk-sac mesoderm cells occurs prior to formation of the primitive streak, diminishing the likelihood that yolk-sac mesoderm cells arise from the epiblast. Morphologic evidence supporting the endodermal derivation of yolk-sac mesoderm includes the cell-to-cell communication displayed by endoderm and mesoderm cells. Furthermore, some yolksac endoderm cells display extensive endoplasmic reticulum and extracellular matrix proteins production that is characteristics of yolk-sac mesoderm [90].

The first stage of yolk-sac formation ends as the primary yolk sac appears to undergo collapse with heterogenous foci of reexpansion leading to the breaking up of the primary 
yolk sac into smaller vesicles (Fig. 4). The secondary yolk sac is subsequently formed at days $12-15$ from the remnants of the primary yolk sac (Fig. 4). The secondary yolk sac begins as a small structure with a diameter measuring less than $0.4 \mathrm{~mm}$ but steadily increases in size to $2 \mathrm{~mm}$ by day 19 , 4-5 $\mathrm{mm}$ by week 7, and 6-6.5 $\mathrm{mm}$ by the end of week 10 .

The first yolk-sac blood islands are evident morphologically in the secondary yolk sac beginning at approximately day 18 of gestation [91,92]. The developmental origin of blood cells and endothelial cells that make up the blood islands is also thought to be the endoderm of the yolk sac.
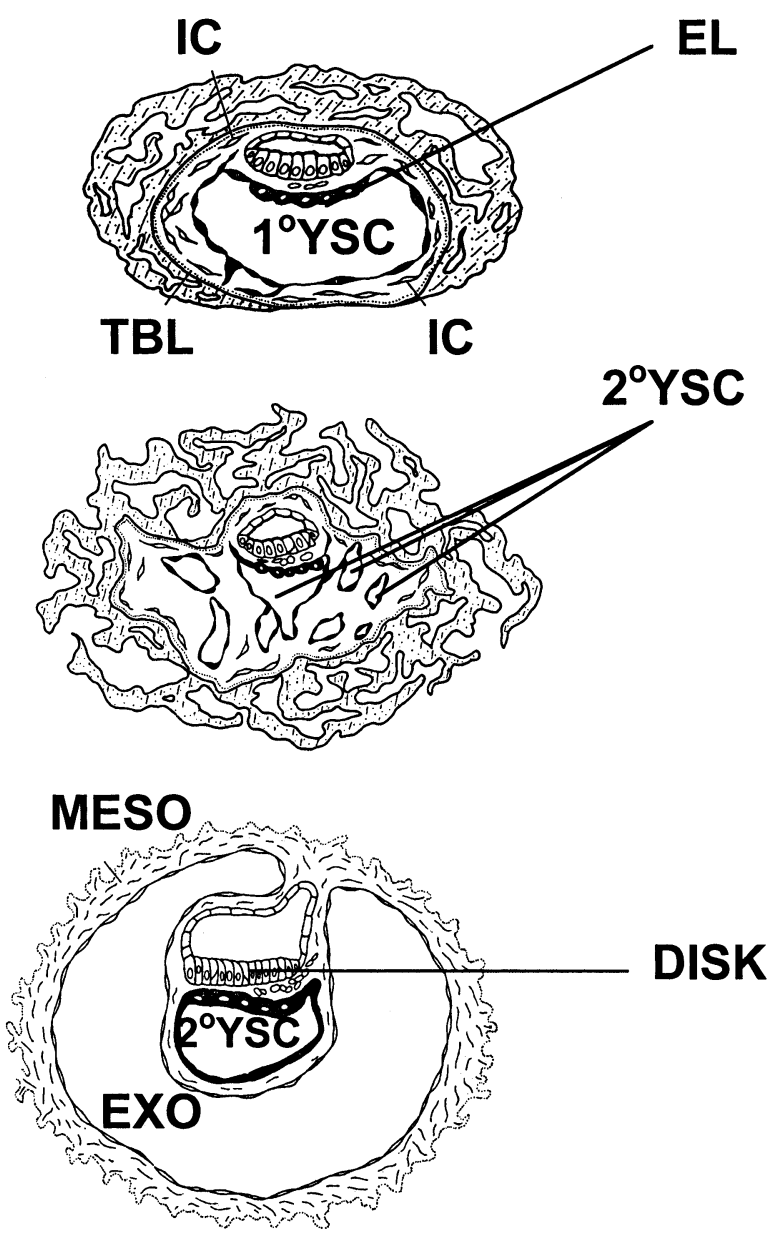

Figure 4. Stages of human yolk sac formation. (Top) The primary yolk sac $\left(1^{\circ} \mathrm{YSC}\right)$ is formed $7-8$ days postconception. Intermediate cells (IC; depicted as white cells) migrate from the endodermal (EL) layer (dark-colored cells) of the primary yolk sac to fill in the space between the endoderm and trophoblastic basal lamina (TBL). (Middle) The secondary yolk sac $\left(2^{\circ} \mathrm{YSC}\right)$ is formed by the remnants of the primary yolk sac following its collapse and fragmentation. (Bottom) By 12 days postconception, the secondary yolk sac has expanded to the edge of the embryonic disk, is lined by endoderm cells (dark cells), and protrudes into the exocelom (EXO). The mesoderm adjacent to the trophoblastic basal lamina (TBL) has differentiated into mesothelium (MESO). Hematopoietic activity will be present in yolk sac blood islands arising in the secondary yolk sac by week 3 of gestation. Reproduced with permission of F.F. Nogales (ed): The Human Yolk Sac and Yolk Sac Tumors, Berlin: Springer-Verlag, 1993, pp. 35-36.
Takashina [93] has provided extensive light and electron microscopic evidence suggesting that blood cells arise in the yolk-sac endoderm and migrate into surrounding yolksac mesenchyme. These blood cells become surrounded by other endoderm-derived mesoderm cells that differentiate into endothelial cells and these blood cell-endothelial cell aggregates develop into blood islands. The morphologic studies supporting the origin of human yolk-sac blood islands from the hypoblast are in striking contrast to studies in rodents and birds that indicate blood cells in the yolk sac are derived from the epiblast.

Besides hematopoiesis, the secondary yolk sac is an active site of protein biosynthesis and nutrient transport $[90,94]$. The endodermal component of the primate secondary yolk sac is a known transporter of proteins and vitamins [95-97]. As the yolk sac matures, an extensive network of superficial capillaries is formed in the mesenchymal layer. The secondary yolk-sac circulation is fed and drained by the vitelline (omphalomesenteric) arteries and veins that connect the embryonic to the extraembryonic circulation. Initially, the vitelline vessels empty into the sinus venosus of the liver, establishing a preportal-type circulation.

Unlike the yolk sac of the mouse that functions throughout gestation, the yolk sac of the human begins regressing around week 10 of gestation. Over the ensuing weeks, there is progressive evidence of tissue degeneration with loss of blood flow through the yolk-sac vascular network [90]. As the yolk sac regresses, new capillaries arising from the developing gut contribute to the vitelline circulation, and as the liver cords proliferate, the portal circulation is established [98].

\section{Primitive erythropoiesis originates in the yolk sac}

The most extensive morphologic description of human yolk-sac hematopoiesis was reported by Kelemen et al. [45]. Hematopoietic cells were first observed in the mesenchymal layer of the secondary yolk sac at day 18 postconception. Most blood islands were comprised of hematopoietic cells completely surrounded by endothelial cells; however, some extravascular free aggregates of blood cells were present in the mesenchyme in close proximity to the visceral endoderm. The 3-4-week embryo had more defined vessels than the yolk sac, but many vessels were void of hematopoietic cells. At the sixth week of development, both intraembryonic and extraembryonic blood vessels became macroscopically visible and primitive erythroblasts could be identified in the yolk-sac vessels. An overall decline in hematopoiesis was observed in the human yolk sac after the eighth week of gestation.

Primitive erythroblasts constituted nearly $100 \%$ of all nucleated cells in the yolk-sac circulation from the fourth to the fifth week of gestation. By the eighth week, primitive erythroblasts remained the predominant (88\%) circulating blood cell but liver-derived definitive red cells were now evident. In the study by Kelemen et al. [85], no primitive erythroblasts were observed in peripheral blood smears at 
12 weeks gestation. The only other mature blood cell present in the yolk sac was the macrophage. In contrast to normal fetal liver and bone marrow patterns of differentiation, macrophages in the yolk sac arose without evidence of a monocytic precursor.

\section{Definitive hematopoietic \\ progenitors are produced in the yolk sac}

Unlike the mouse, there have been no studies of primitive erythroid progenitors in the human. Huyhn et al. [99] isolated yolk sac, liver, and embryonic tissues from day 25-40 gestation embryos and plated the enzyme disaggregated samples in methylcellulose culture for detection of hematopoietic progenitor cells. While the concentration of BFU-E in the yolk sac and embryo proper were similar, sevenfold to 12-fold higher numbers of CFU-GM were present in the embryo proper compared to the yolk sac. Migliaccio et al. [100] examined the kinetics of definitive hematopoietic progenitors in human yolk sacs from 4.5 to 10 weeks gestation. This stage encompassed the period of yolk sac hematopoietic decline and initiation of liver hematopoiesis. At 4.5 weeks of gestation, BFU-E, CFU-E, and CFU-GM were abundant in the yolk sac, but the concentration of these progenitors subsequently fell precipitously. By 6 weeks of gestation, hematopoietic progenitors were readily detectable in the liver but the yolk sac was devoid of all but a few CFU-E. Over the next three weeks, the concentration of BFU-E, CFU-E, and CFU-GM rose rapidly in the liver and reached a plateau by $8-9$ weeks of gestation. In addition, a gradual shift from primarily embryonic to primarily fetal and adult hemoglobin molecules occurs in red blood cells derived from yolk sac and liver BFU-E [101], during the fifth to eighth weeks of gestation. These results suggest that the yolk sac serves as the source of the first definitive erythroid and macrophage progenitors in the human embryo, similar to recent murine studies (above). However, a detailed analysis of embryonic tissues prior to the onset of circulation has not been conducted to confirm this association.

\section{Multipotential hematopoietic \\ progenitors are produced in the yolk sac}

HPP-CFC have been localized to both the yolk sac and embryo proper of the day 35-40 gestation embryo cells [99]. $\mathrm{CD} 34^{+}$hematopoietic cells recovered from the yolk sac and embryo yielded 45 HPP-CFC and 20 HPP-CFC, respectively, when $10,000 \mathrm{CD}^{+} 4^{+}$cells were plated with the growth factors recombinant human stem cell factor (rhuSCF) and interleukin-3 (IL-3). Addition of recombinant human granulocyte (rhuGCSF) or granulocyte-macrophage (rhuGM-CSF) colony-stimulating factors failed to influence the number or size of the HPP-CFC grown in rhuSCF or rhuIL-3 [99]. All CD $34^{+}$progenitors and HPP-CFC present in the yolk sac and embryo proper failed to express CD38 or CD33, consistent with the pattern of expression observed for early hematopoietic precursors in the adult marrow.

\section{Summary}

We have reviewed the existing data regarding the ontogeny of hematopoiesis in the human yolk sac. As in the mouse, the yolk sac in humans gives rise to differentiating primitive erythroblasts and macrophage cells. At the progenitor level, investigation has been limited to definitive hematopoietic lineages, but the results are most congruent with the yolk sac serving as the first site of BFU-E, CFU-E, and MacCFC synthesis. Analysis of the ability of yolk sac cells to engraft in vivo has not been conducted in part because such transplant studies are untenable in human patients. The recent description of human/mouse xenotransplant models for the identification of human SCID-repopulating stem cells may now make these studies feasible. If embryonic HSCs exist in the human embryonic yolk sac, these cells may only be detectable upon transplantation into fetal or newborn rather than adult NOD/SCID mice. The underlying biological behavior of such embryonic HSCs would need to be investigated before the use of yolk-sac cells for human transplantation could be envisioned.

\section{References}

1. Moore MAS, Owen JJT (1967) Stem-cell migration in developing myeloid and lymphoid systems. Lancet i:658

2. Keller G, Lacaud G, Robertson S (1999) Development of the hematopoietic system in the mouse. Exp Hematol 27:777

3. Jollie WP (1990) Development, morphology, and function of the yolk-sac placenta of laboratory rodents. Teratology 41:361

4. Miura Y, Wilt FH (1969) Tissue interaction and the formation of the first erythroblasts of the chick embryo. Dev Biol 19:201

5. Belaousoff M, Farrington SM, Baron MH (1999) Hematopoietic induction and respecification of A-P identity by visceral endoderm signaling in the mouse embryo. Development 125:5009

6. Palis J, McGrath KE, Kingsley PD (1995) Initiation of hematopoiesis and vasculogenesis in murine yolk sac explants. Blood 86:156

7. Bielinska M, Narita N, Heikinheimo M, et al. (1996) Erythropoiesis and vasculogenesis in embryoid bodies lacking visceral yolk sac endoderm. Blood 88:3720

8. Maximow AA (1909) Untersuchungen uber blut und bindegewebe 1 . Die fruhesten entwicklungsstadien der blut und bindegewebszellan bein saugeberembryo, bis zum anfang der blutbilding unden leber. Arch Mikroskop Anat 73:444

9. Haar JL, Ackerman GA (1971) A phase and electron microscopic study of vasculogenesis and erythropoiesis in the yolk sac of the mouse. Anat Rec 170:199

10. Sasaki K, Matsamura G (1986) Haemopoietic cells of yolk sac and liver in the mouse embryo: a light and electron microscopical study. $\mathrm{J}$ Anat 148:87

11. Eichmann A, Corbel C, Nataf V, et al. (1997) Ligand-dependent development of the endothelial and hemopoietic lineages from embryonic mesoderm cells expressing vascular endothelial growth factor receptor 2. Proc Natl Acad Sci U S A 94:5141

12. Choi K, Kennedy M, Kazarov A, et al. (1998) A common precursor for hematopoietic and endothelial cells. Development 125:725

13. Shalaby F, Rossant J, Yamaguchi TP, et al. (1995) Failure of bloodisland formation and vasculogenesis in Flk-1-deficient mice. Nature 376:62

14. Bethlenfalvay NC, Block M (1970) Fetal erythropoiesis. Maturation in megaloblastic (yolk sac) erythropoiesis in the C57B1/6J mouse. Acta Haemat 44:240 
15. de la Chapelle A, Fantoni A, Marks P (1969) Differentiation of mammalian somatic cells: DNA and hemoglobin synthesis in fetal mouse yolk sac erythroid cells. Proc Natl Acad Sci U S A 63:812

16. Sangiorgi F, Woods CM, Lazarides E (1990) Vimentin downregulation is an inherent feature of murine erythropoiesis and occurs independently of lineage. Development 110:85

17. Fantoni A, de la Chapelle A, Rifkind RA, et al. (1968) Erythroid cell development in fetal mice: synthetic capacity for different proteins. J Mol Biol 33:79

18. Boussios T, Bertles JF (1988) The globin gene expression program in the hamster embryo. Exp Hematol 16:1

19. Steiner R, Vogel H (1973) On the kinetics of erythroid cell differentiation in fetal mice: I. Microspectrophotometric determination of the hemoglobin content in erythroid cells during gestation. J Cell Physiol $81: 323$

20. Barker JE (1968) Development of the mouse hematopoietic system: I. Types of hemoglobin produced in embryonic yolk sac and liver. Dev Biol 18:14

21. Brotherton TW, Chui DHK, Gauldie J, et al. (1979) Hemoglobin ontogeny during normal mouse fetal development. Proc Natl Acad Sci U S A 76:2853

22. Chui DHK, Loyer BV (1975) Foetal erythropoiesis in Steel mutant mice. II. Haemopoietic stem cells in foetal livers during development. Br J Haematol 29:553

23. Kaufman MH (1992) The atlas of mouse development. New York: Academic Press

24. Morioka K, Tone S, Mukaida M, et al. (1998) The apoptotic and nonapoptotic nature of the terminal differentiation of erythroid cells. Exp Cell Res 240:206

25. Koury M, Bondurant M (1992) The molecular mechanism of erythropoietin action. Eur J Biochem 210:649

26. Cole RJ, Paul J (1966) The effects of erythropoietin on haem synthesis in mouse yolk sac and cultured foetal liver cells. J Emb Exp Morph 15:245

27. Bateman AE, Cole RJ (1971) Stimulation of haem synthesis by erythropoietin in mouse yolk-sac-stage embryonic cells. J Emb Exp Morph 26:475

28. Kimura T, Sonoda Y, Iwai N, et al. (2000) Proliferation and cell death of embryonic primitive erythrocytes. Exp Hematol 28:635

29. Wong PMC, Chung S-H, Reicheld SM, et al. (1986) Hemoglobin switching during murine embryonic development: evidence for two populations of embryonic erythropoietic progenitor cells. Blood 67:716

30. McGann JK, Silver L, Liesveld J, et al. (1997) Erythropoietin-receptor expression and function during the initiation of murine yolk sac erythropoiesis. Exp Hematol 25:1149

31. Wu H, Liu X, Jaenisch R, et al. (1995) Generation of committed erythroid BFU-E and CFU-E progenitors does not require erythropoietin or the erythropoietin receptor. Cell 83:59

32. Kieran MW, Perkins AC, Orkin SH, et al. (1996) Thrombopoietin rescues in vitro erythroid colony formation from mouse embryos lacking the erythropoietin receptor. Proc Natl Acad Sci U S A 93:9126

33. Lin C-S, Lim S-K, D'Agati V, et al. (1996) Differential effects of an erythropoietin receptor gene disruption on primitive and definitive erythropoiesis. Genes Dev 10:154

34. Neubauer H, Cumano A, Muller M, et al. (1998) Jak2 deficiency defines an essential developmental checkpoint in definitive hematopoiesis. Cell 93:397

35. Boussios T, Bertles JF, Goldwasser E (1989) Erythropoietin-receptor characteristics during the ontogeny of hamster yolk sac erythroid cells. J Biol Chem 264:16017

36. Harrison PR, Conkie D, Affara N, et al. (1974) In situ localization of globin messemger RNA formation. I. During mouse fetal liver development. J Cell Biol 63:402

37. Koury ST, Bondurant MC, Koury MJ, et al. (1991) Localization of cells producing erythropoietin in murine liver by in situ hybridization. Blood 77:2497
38. Keller G, Kennedy M, Papayannopoulou T, et al. (1993) Hematopoietic commitment during embryonic stem cell differentiation in culture. Mol Cell Biol 13:473

39. Koury MJ, Bondurant MC, Graber SE, et al. (1988) Erythropoietin messenger RNA levels in developing mice and transfer of 125Ierythropoietin by the placenta. J Clin Invest 82:154

40. Wong PMC, Chung SW, Chui DHK, et al. (1986) Properties of the earliest clonogenic hematopoietic precursors to appear in the developing murine yolk sac. Proc Natl Acad Sci U S A 83:3851

41. Kennedy M, Firpo M, Choi K, et al. (1997) Identification of a common precursor for primitive and definitive hematopoiesis. Nature 386:488

42. Palis J, Robertson S, Kennedy M, et al. (1999) Development of erythroid and myeloid progenitors in the yolk sac and embryo proper of the mouse. Development 126:5073

43. Johnson GR, Barker DC (1985) Erythroid progenitor cells and stimulating factors during murine embryonic and fetal development. Exp Hematol 13:200

44. Wong PMC, Clarke BJ, Carr DH, et al. (1982) Adult hemoglobins are synthesized in erythroid colonies in vitro derived from murine circulating hemopoietic progenitor cells during embryonic development. Proc Natl Acad Sci U S A 79:2952

45. Cudennec CA, Thiery J-P, Le Douarin NM (1981) In vitro induction of adult erythropoiesis in early mouse yolk sac. Proc Natl Acad Sci U S A 78:2412

46. Lichanska AM, Hume DA (2000) Origins and functions of phagocytes in the embryo. Exp Hematol 29:601

47. Takahashi K, Yamamura F, Naito M (1989) Differentiation, maturation, and proliferation of macrophages in the mouse yolk sac: A lightmicroscopic, enzyme-cytochemical, immunohistochemical, and ultrastructural study. J Leukoc Biol 45:87

48. Morris L, Graham CF, Gordon S (1991) Macrophages in haemopoietic and other tissues of the developing mouse detected by the monoclonal antibody F4/80. Development 112:517

49. Hume DA, Monkley SJ, Wainwright BJ (1995) Detection of c-fms protooncogene in early mouse embryos by whole mount in situ hybridization indicates roles for macrophages in tissue remodelling. $\mathrm{Br}$ J Haematol 90:939

50. Moore MAS, Metcalf D (1970) Ontogeny of the haemopoietic system: yolk sac origin in vivo and in vitro colony forming cells in the developing mouse embryo. Br J Haematol 18:279

51. Alliot F, Godin I, Pessac B (1999) Microglia derive from progenitors, originating from the yolk sac, and which proliferate in the brain. Brain Res Dev Brain Res 117:145

52. Kurz H, Christ B (1998) Embryonic CNS macrophages and microglia do not stem from circulating, but from extravascular precursors. Glia 22:98

53. Sonoda T, Hayashi C, Kitamura Y (1983) Presence of mast cell precursors in the yolk sac of mice. Dev Biol 97:89

54. Bertoncello I (1992) Status of high proliferative potential colonyforming cells in the hematopoietic stem cell hierarchy. Curr Top Microbiol Immunol 177:83

55. Medvinsky AL, Samoylina NL, Muller AM, et al. (1993) An early pre-liver intra-embryonic source of CFU-S in the developing mouse. Nature 364:64

56. Yoder MC, Hiatt K (1999) Murine yolk sac and bone marrow hematopoietic cells with high proliferative potential display different capacities for producing colony-forming cells ex vivo. J Hematother Stem Cell Res 8:421

57. Palis J, Chan RJ, Koniski A, et al. (2001) Spatial and temporal emergence of high proliferative potential hematopoietic precursors during murine embryogenesis. Proc Natl Acad Sci U S A 98:4528

58. Tyan ML, Herzenberg LA (1968) Studies of the ontogeny of the mouse immune system II. Immunoglobulin-producing cells. J Immunol 101:446

59. Cumano A, Furlonger C, Paige CJ (1993) Differentiation and characterization of B-cell precursors detected in the yolk sac and embryo 
body of embryos beginning at the 10- to 12 -somite stage. Proc Natl Acad Sci U S A 90:6429

60. Huang H, Zettergren LD, Auerbach R (1994) In vitro differentiation of $\mathrm{B}$ cells and myeloid cells from the early mouse embryo and its extraembryonic yolk sac. Exp Hematol 22:19

61. Godin I, Dieterlen-Lievre F, Cumano A (1995) Emergence of multipotent hemopoietic cells in the yolk sac and paraaortic splanchnopleura in mouse embryos, beginning at 8.5 days postcoitus. Proc Natl Acad Sci U S A 92:773

62. Ogawa M, Nishikawa S, Ikuta K, et al. (1988) B cell ontogeny in murine embryo studied by a culture system with the monolayer of a stromal cell clone, ST2: B cell progenitor develops first in the embryonal body rather than in the yolk sac. EMBO J 7:1337

63. Godin IE, Garcia-Porrero JA, Coutinho A, et al. (1993) Para-aortic splanchnopleura from early mouse embryos contains B1a cell progenitors. Nature 364:67

64. Cumano A, Dieterlen-Lievre F, Godin I (1996) Lymphoid potential, probed before circulation in mouse, is restricted to caudal intraembryonic splanchnopleura. Cell 86:907

65. Liu C-P, Auerbach R (1991) Ontogeny of murine T cells: thymusregulated development of $\mathrm{T}$ cell receptor-bearing cells derived from embryonic yolk sac. Eur J Immunol 21:1849

66. Ohmura K, Kawamoto H, Fujimoto S, et al. (1999) Emergence of T, $\mathrm{B}$, and myeloid lineage-committed as well as multipotent hemopoietic progenitors in the aorta-gonad-mesonephros region of day $10 \mathrm{fe}-$ tuses of the mouse. J Immunol 163:4788

67. Harrison DE, Astle CM, DeLaittre JA (1979) Processing by the thymus is not required for cells that cure and populate $\mathrm{W} / \mathrm{Wv}$ recipients. Blood 54:1152

68. Muller AM, Medvinsky A, Strouboulis J, et al. (1994) Development of hematopoietic stem cell activity in the mouse embryo. Immunity 1:291

69. Ema H, Nakauchi H (2000) Expansion of hematopoietic stem cells in the developing liver of the mouse embryo. Blood 95:2284

70. Jordan C, Astle C, Zawadzki J, et al. (1995) Long-term repopulating abilities of enriched fetal liver stem cells measured by competitive repopulation. Exp Hematol 23:1011

71. Sanchez M-J, Holmes A, Miles C, et al. (1996) Characterization of the first hematopoietic stem cells in the AGM and liver of the mouse embryo. Immunity 5:513

72. Houssaint E (1981) Differentiation of the mouse hepatic primordium II. Extrinsic origin of the haemopoietic cell line. Cell Differ 10:243

73. Dieterlen-Lievre F (1992) Embryonic chimeras and hematopoietic system development. Bone Marrow Transplant 9:30

74. Smith RA, Glomski CA (1982) "Hemogenic endothelium" of the embryonic aorta: does it exist? Dev Comp Immunol 6:359

75. Tavian M, Coulombel L, Luton D, et al. (1996) Aorta-associated $\mathrm{CD}^{+} 4^{+}$hematopoietic cells in the early human embryo. Blood 87:67

76. de Bruijn MFTR, Speck NA, Peeters MCE, et al. (2000) Definitive hematopoietic stem cells first develop within the major arterial regions of the mouse embryo. EMBO J 19:2465

77. Toles JF, Chui DHK, Belbeck LW, et al. (1989) Hematopoietic stem cells in murine embryonic yolk sac and peripheral blood. Proc Natl Acad Sci U S A 86:7456

78. Weissman I, Papaioannou V, Gardner R (1978) Fetal hematopoietic origins of the adult hematolymphoid system. Cold Spring Harbor, NY: Cold Spring Harbor Laboratory

79. Fleischman RA, Custer RP, Mintz B (1982) Totipotent hematopoietic stem cells: normal self-renewal and differentiation after transplantation between mouse fetuses. Cell 30:351

80. Yoder M, Cumming JG, Hiatt K, et al. (1996) A novel method of my- eloablation to enhance angraftment of adult bone marrow cells in newborn mice. Biology Blood Marrow Transplantation 2:59

81. Harrison DE, Astle CM (1997) Short- and long-term multilineage repopulating hematopoietic stem cells in late fetal and newborn mice: Models for human umbilical cord blood. Blood 90:174

82. Yoder MC, Hiatt K (1997) Engraftment of embryonic hematopoietic cells in conditioned newborn recipients. Blood 89:2176

83. Yoder MC, Hiatt K, Dutt P, et al. (1997) Characterization of definitive lymphohematopoietic stem cells in the day 9 murine yolk sac. Immunity 7:335

84. Yoder MC, Hiatt K, Mukherjee P (1997) In vivo repopulating hematopoietic stem cells are present in murine yolk sac at day 9.0 postcoitus. Proc Natl Acad Sci U S A 94:6776

85. Kelemen E, Calvo W (1979) Atlas of human hematopoietic development. New York: Springer-Verlag

86. Charbord P, Tavian M, Humeau L, et al. (1996) Early ontogeny of the human marrow from long bones: an immunohistochemical study of hematopoiesis and its microenvironment. Blood 87:4109

87. Kelemen E, Gulya E, Vass K (1978) Ontogeny of human neutrophil granulocyte alkaline phosphatase. J Cell Physiol 93:353

88. Stamatoyannopoulos G, Constantoulakis P, Brice M, et al. (1987) Coexpression of embryonic, fetal, and adult globins in erythroid cells of human embryos: relevance to the cell-lineage models of globin switching. Dev Biol 123:191

89. Tavassoli M (1988) Ontogeny of hemopoiesis. In: Meisami E, Timiras P (eds.): Handbook of human growth and developmental biology. Boca Raton: CRC Press, p. 101

90. Enders AC, King BF (1993) Development of the human yolk sac. In: Nogales FF (ed.) The human yolk sac and yolk sac tumors. Berlin: Springer-Verlag, p. 33

91. Bloom W, Bartelmez GW (1940) Hematopoiesis in young human embryos. Am J Anat 67:21

92. Luckett WP (1978) Origin and differentiation of the yolk sac and extraembryonic mesoderm in presomite human and rhesus monkey embryos. Am J Anat 152:59

93. Takashina T (1987) Haemopoiesis in the human yolk sac. J Anat 151:125

94. Azoulay MC, Webb CG, Sach L (1987) Control of hematopoietic cell growth regulators during mouse fetal development. Mol Cell Biol $7: 3361$

95. Wilson J, King B (1985) Transport of horseradish peroxidase across monkey trophoblast epithelium in coated and uncoated vesicles. Anat Rec 211:174

96. Brent RL, Beckman DA, Jensen M, et al. (1990) Experimental yolk sac dysfunction as a model for studying the nutritional disturbances in the embryo during early organogenesis. Teratology 41:405

97. Brent RL, Fawcett L (1998) Nutritional studies of the embryo during early organogenesis with normal embryos and embryos exhibiting yolk sac dysfunction. J Pediatr 132:S6

98. Bremer J (1914) The earliest blood vessels in man. Am J Anat 16:447

99. Huyhn A, Dommergues M, Izac B, et al. (1995) Characterization of hematopoietic progenitors from human yolk sacs and embryos. Blood $86: 4474$

100. Migliaccio G, Migliaccio AR, Petti S, et al. (1986) Human embryonic hematopoiesis: kinetics of progenitors and precursors underlying the yolk sac to liver transition. J Clin Invest 78:51

101. Peschle C, Migliaccio AR, Migliaccio G, et al. (1984) Embryonic $\rightarrow$ fetal $\mathrm{Hb}$ switch in humans: Studies on erythroid bursts generated by embryonic progenitors from yolk sac and liver. Proc Natl Acad Sci U S A 81:2416 